\title{
TOWARDS A PORTABLE, MEMORY-EFFICIENT TEST SYSTEM FOR CONDUCTED ENERGY WEAPONS
}

\author{
Peyman Rahmati ${ }^{1}$, David Dawson ${ }^{1}$, and Andy Adler ${ }^{1}$ \\ ${ }^{1}$ Carleton University, Ottawa, Canada. \{prahmati,adler\}@ sce.carleton.ca
}

\begin{abstract}
We present a readily portable, memory-efficient performance test system (PTS) for Tasers. The proposed PTS has been developped for the most widely used Conducted Energy Weapons (CEW), Taser X26. The PTS is designed in accordance with the CEW Test Procedure, recently adopted and published by a group of experts. This work is an advancement of our earlier work where we developed a performance calibration system for the Taser X26 in 2010. The objective of the proposed PTS is to check whether the electrical specifications of the Taser $\mathrm{X} 26$, listed in the CEW test procedure, fall within the manufacturers limits for satisfactory electrical performance or not. Additionally data above and beyond that necessary for performance validation is generated for further study of failure modes and biomedical effects. A new data file format is proposed to create a consistent structure for a data repository and data mining in future research. The CEW is electrically connected to a calibrated dummy resistive network of $600 \mathrm{ohms}$ and fired for 5 seconds while the output voltage is captured with a sampling rate of $10 \mathrm{MS} / \mathrm{s}$ at 12 bit resolution. In comparison with our earlier PTS, this one is faster, of higher resolution and higher accuracy, mobile, and memory-efficient.
\end{abstract}

Index Terms - Conducted Energy Weapons, Calibration, Electrical Stimulation, Electrophysiological Standards

\section{INTRODUCTION}

Conducted Energy Weapons (CEWs) use electrical stimulation to cause neuromuscular incapacitation [1]. The most common CEW in use with police forces is the Taser X26. However, since CEW use has been associated with some high profile deaths, there is considerable controversy over their safety, effectiveness and associated usage policy. Until very recently in Canada, there was no benchmark or regular testing of these weapons for conformance with performance specifications. As a result of government policy some jurisdictions have directed that all police services will have these weapons tested regularly, usually on an annual basis. Other jurisdictions have taken a wait-and-see attitude while still others have adopted a commendable pro-active approach to performance verification and lifecycle management. The public interest, while focussing primarily on the biomedical effect of CEW's, has heightened interest in all aspects of weapons management.

In an earlier paper we described the creation of a test system for validating the performance of CEW's in accordance with the manufacturer's performance standards [2]. Since that time government, industry and academia have collaborated to create and promulgate a test procotol, proposed by a group of Canadian experts, for all CEW's which incorporates not only the performance parameters set out by the manufacturer but provides a data set and bridge to safety standards for these weapons. The CEWs are restricted weapons described in the Criminal Code of Canada [3]. No one except sworn police officers or specially authorized entities may possess and store CEW's. Therefore, it is helpful to provide an in-situ testing regime which leaves weapons in their own environment at the premises of the police service and obviates the need for shipping, handling and delay in reporting test results. Our earlier work in [2] describes a test setup that permitted in-situ testing of weapons obviating the need for shipping, handling and extensive delay in testing large numbers of weapons.

In this paper we describe a new Portable Test System (PTS) for Conducted Energy Weapons which is more mobile, more memory efficient, more comprehensive, and fully conformant with the Canadian Test Protocol for CEW's. Moreover, the designed test system produces a new data file format which establishes an economical and consistent structure for performance data as well as enabling data mining in the future.

The remainder of the paper is organized as follows: the definition of electrical parameters to characterize the electrical performance of a CEW is presented in the next section. Then the design development, experimental setup and the proposed file format to store the data set in memory are presented. Section 4 discusses the tabulated results. Finally we draw conclusions and discussion in section 5 .

\section{ELECTRICAL SPECIFICATIONS OF THE X-26 AND THE BIOMEDICAL EFFECT}

The Taser X26 fires a sequence of pulses (pulse train) at a typical rate of 20 pulses per second. Attaching the weapon output terminals to a target, it produces either a closed circuit with high conducted current or an open circuit with low conducted current. 


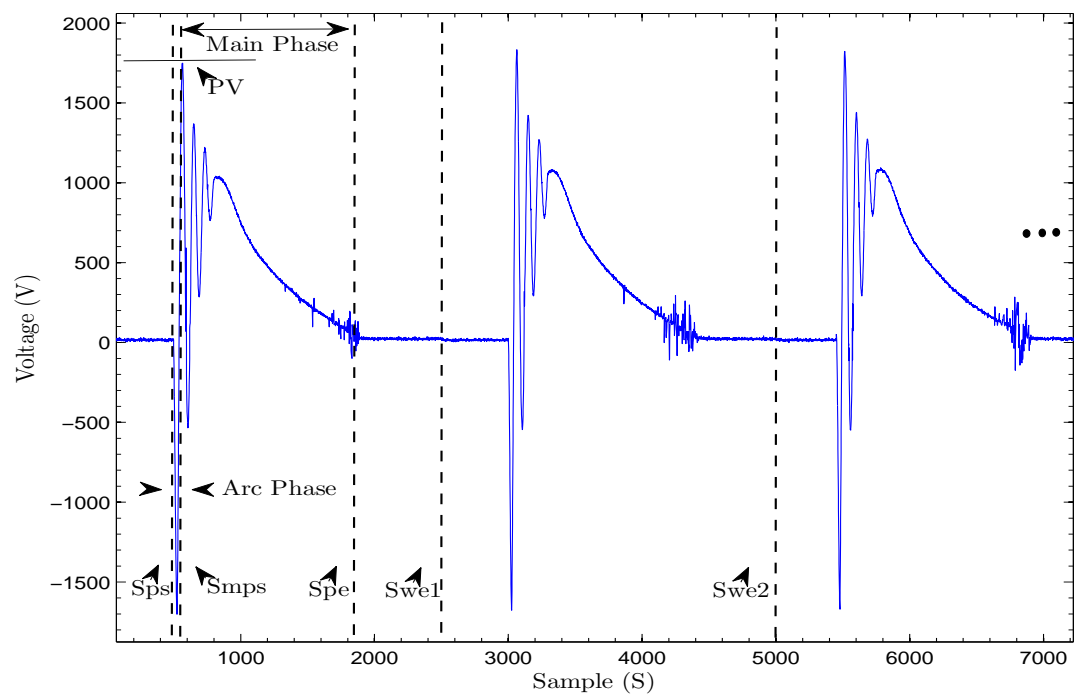

Fig. 1. The first three pulses of the Taser X26 representing the gun electrical parameters as well as pulse windowing applied for the Pico 4224.

The closed circuit occurs when resistive path between the weapon output terminals is low while it is a high resistive path for an open circuit. Fig. 1 depicts the first three pulses of the pulse train for the Taser X26. The Taser X26 mainly utilizes three different capacitors to produce the pulse train. A single pulse of the Taser X26 is produced by charging and discharging the three capacitors with three different time constants, producing three different frequencies in the output waveform. There are two phases for a single pulse of the Taser X26: Arc Phase and Main Phase. The Arc Phase is the highest frequency part of the pulse involving the rapid pulse with the highest negative peak, conducting a very high current to the target at the firing moment. The Main Phase delivers the main part of the energy and the charge to the target. In 2009, six electrical parameters were selected to be measured for taser performance monitoring [4]. It was agreed to at the 2010 CEW workshop [5] to provide the graphical definition of these parameters as well as that of some currently added ones. The six parameters are [4]: 1) Peak Voltage (PV) which is peak of main phase voltage on a pulse; 2) Peak Current (PC) which is peak of main phase current over a pulse; 3) Full Charge (FC) representing the integral of the absolute value of the current over the entire pulse (arc phase + main phase); 4) Net Charge (NC) showing the integral of the main phase current on a pulse; 5) Pulse duration (PD) which is the time from starting point of a waveform at an initial crossing of $\pm 50 \mathrm{~V}$ to end point at a pulse decrement to $\pm 50 \mathrm{~V}$ on a pulse, given, in fig. 1, the start point as:

$$
t_{p s}=\frac{S_{p s}}{f_{s}}
$$

where $S_{p s}$ is the sample at the starting point of a waveform and $f_{s}$ is the sampling rate. And,

$$
t_{p e}=\frac{S_{p e}}{f_{s}},
$$

where $S_{p e}$ is the sample at the end point of a waveform.
6) Interpulse time (IPT) which is the time from $t_{p s}$ of one pulse to $t_{p s}$ of the next pulse. Pulse repetition frequency (PRF) or number of pulses per second (pps) is another added parameter in [4] defined as:

$$
P R F=\frac{N_{p}-1}{T_{f}}=\frac{1}{\text { Average IPT }},
$$

where $N_{p}$ is number of pulses and $T_{f}$ is firing-length time (or equivalently total pulse train duration).

The international standard represented in $[6,7]$ offers how to summarize the measured electrical parameters of each individual pulse in a pulse train.

The risk of CEW's has been studied from many different points of view: electrical safety $[8,9]$, animal applications $[10,11,9]$, and human tests [12]. The determination of the six electrical parameters mentioned above as well as the PRF may be useful to test against safety limits developed by future electrophysiological research [13]. One standard to assess the ventricular fibrillation risk, IEC 60479 Part 2, has been referenced in [14], which defines curves based on "the probability of fibrillation risk for current flowing through the body from the left hand to both feet" [14]. It considers the current signals to be "unidirectional single impulse currents of short durations" [14]. We base our calculation on the "C1 curve" which is defined as "no risk of brillation". The standard proposes that to have "no risk of fibrillation", the cumulative charge of the single current pulse of $100 \mu$ s should not be higher than a safety limit of $710 \mu \mathrm{C}$ [14].

Monophasic Charge (MC), which is the maximum of the integral of current in either direction in a pulse, has been proposed as an appropriate measure to test against the safety limit $(710 \mu \mathrm{C})$ for the Taser X26 [13]. This is because taser pulses are not unidirectional as it is supposed in IEC (2007) and MC "may be justified based on physiological models" such as Reilly et al (2009) [13]. 


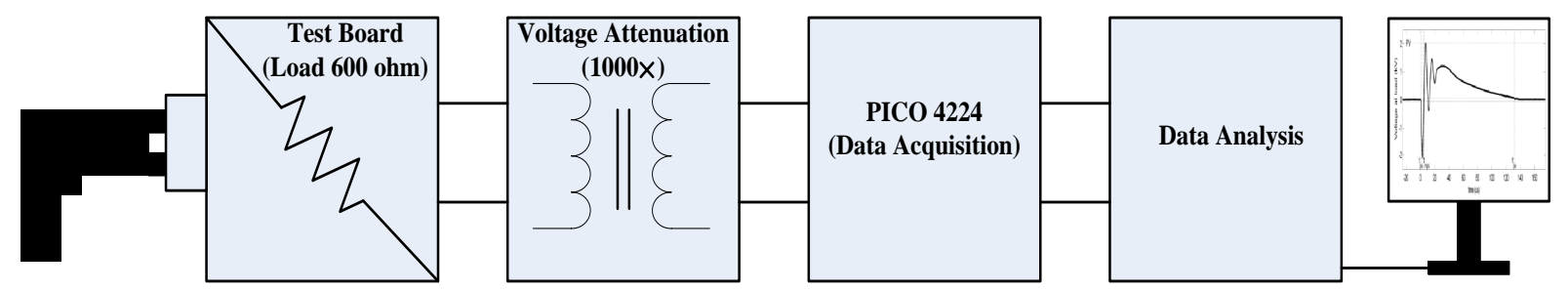

Fig. 2. Overview of the developed TPS representing different blocks of the setup.

J. P. Reilly et al in [15] investigated the biomedical effect of CEWs in a different manner by proposing a threshold factor named $F_{T}$ to simulate the stimulation of a myelinated reference-case neuron ( $20 \mu \mathrm{m}$ diameter, $1 \mathrm{~cm}$ distant). An $F_{T}$ of unity means a single action potential (AP) has been propagated into the reference-case neuron. The stimulus under the $F_{T}$ does not generate any AP in the peripheral neurons (No incapacitation of the target). This work shows different CEWs have different $F_{T}$ to stimulate the reference-case neuron. The electrical parameters of different CEWs have been measured under the same condition of having an excitation level of unity $\left(F_{T}=1\right)$, meaning that the neurons surronding the stimulation electrode are excited for all CEWs under investigation. According to the experimental data, the $F_{T}$ for CEWs decreases as the resistive load increases [15]. For the Taser X26 application, we consider a test resistive load of 600 ohms, recommended by TI, to be an adequate model of the impedance load of the body.

\section{DESIGN OF TASER PTS AND EXPERIMENTAL SETUP}

The developed PTS has four distinct parts represented by the block diagram in fig. 2 - the energy source (Taser X-26), the data acquisition block, the analysis block, and monitoring block. The data acquisition block consists of a high-resolution data acquisition system (Pico 4224, Pico Technology Company, UK), calibrated high voltage probe, a calibrated dummy resistive network of $600 \mathrm{ohms}$ embedded in a test board, fig. 2 . The analysis block processes the raw data acquired from the weapon to monitor the electrical parameters and to validate the performance of the weapon in accordance with the ranges set out in the manufacturer's performance specifications. The weapon is electrically connected to the calibrated purely resistive load of $600 \mathrm{ohms}$. The sampling rate is set up to 10 $\mathrm{MS} / \mathrm{s}$ with a resolution of 12 bits. We ran the data acquisition system in a memory-efficient manner using "rapid block mode". The data acquisition system in this mode is designed to sample the taser pulse train only where the energy is delivered to the target. If the input analog voltage signal is bigger than a predefined threshold level, the signal will be sampled over a time window. The length of the window should be big enough to involve the maximum possible length of a single pulse. In our experiments, the length is set to be 2500 sam- ples (or equivalently $250 \mu \mathrm{s}=\frac{2500 \text { samples }}{\text { Sampling rate }(10 \mathrm{MHz})}$ ). The data acquisition system captures the data $50 \mu$ s before the trigger event, set at $1000 \mathrm{~V}$, and $200 \mu$ s after, constructing a $250 \mu$ s window. Fig. 1 shows the end of the first window by $S_{w e 1}$ for the first captured pulse and by $S_{w e 2}$ for the second one.

Fig. 3 shows the proposed taser data file format involving three different data types: test specifications, IPT data, and taser sampling data. The test specifications section in the data file format contains the following information: Software version, measurement time, serial number of the weapon, description, owner's name, comments, gain of data acquisition system, offset of data acquisition system, resistance of applied load, sampling rate, number of collected pulses, and pulse length. The timing data involves the IPTs for the collected pulse train, and the raw data is the sampled and quantized voltage measurement when the weapon is emitting energy, see fig. 3.

Using the proposed test system, a sufficient signal can be acquired in less than 15 seconds (including physical placement of a weapon in the mount, recording serial number, owner's name, and battery capacity) and the analysis is done in about 6 seconds per weapon. Using the summary result table produced at the end of each shot, the protocol instantaneously identifies poorly performing weapons which may require service. This procedure and testing equipment do not duplicate the test equipment and procedure suggested by the manufacturer but it provides sufficient information to determine performance in accordance with the upper and lower performance limits on the parameters established by the manufacturer. Applying a high sampling rate of $10 \mathrm{MS} / \mathrm{s}$ with high resolution of 12 bits, the absolute maxima and minima and the actual pulse shape consisting details can be observed on the final graphical window, see fig. 1 .

\section{RESULTS}

The developed PTS subsequently sampled the signals from 91 different weapons at a rate of $10 \mathrm{MHz}$ with 12 bits resolution using the Pico 4224 through a calibrated high voltage probe. To test performance of the weapon against the parameters set out by the manufacturer, an average of the last 8 pulses was used for analysis. However, a stripped data set in accordance with the proposed data file format in section 3 was recorded from the approximately 5 second discharges (80-100 pulses). The average size of the stripped data set was $410 \mathrm{~KB}$, which 


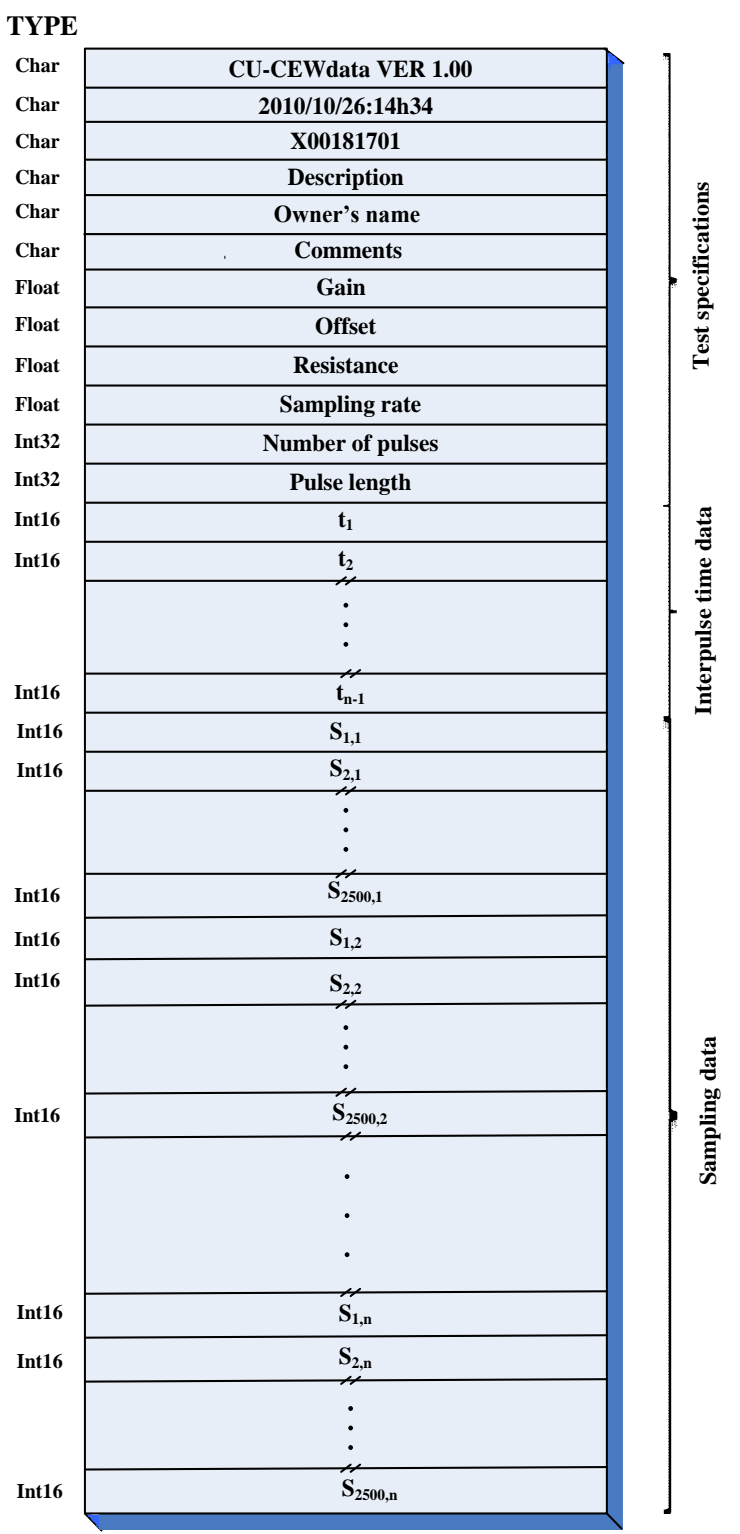

Fig. 3. Representation of the proposed file format for CEWs using memory map.

is a small data file as we are only sampling where the weapon delivers a high energy to the test load.

The proposed PTS was able to successfully identify nonperforming weapons. Over 300 raw data files from 91 different weapons were analyzed to determine overall out-oftolerance rates. The rapid rate of testing, 20 seconds on average to acquire and analyze the data, allowed us to re-test the weapons using new Digital Power Magazines (DPM) where an initial out-of-tolerance condition had been reported. The objective of re-testing was to check whether the out-of-tolerance condition occurs due to the battery malfunction or the weapon itself. Table 1 shows the rates of out-of-tolerance conditions on first, second, and thrid shots. The rate of out-of-tolerance on the first shots was $16.5 \%$, vs. $26.7 \%$ on the second shots and $25 \%$ on the third shots.

Table 1. Results from testing with PicoScope (Note: The outof-tolerance weapons are marked by an asteroid.)

\begin{tabular}{|c|c|c|c|c|}
\hline \multirow[t]{2}{*}{$\begin{array}{l}\text { The number of tested } \\
\text { /re-tested weapons }\end{array}$} & \multicolumn{2}{|l|}{$\begin{array}{c}\text { In } \\
\text { tolerance }\end{array}$} & \multicolumn{2}{|l|}{$\begin{array}{l}\text { Out of } \\
\text { tolerance }\end{array}$} \\
\hline & $\begin{array}{l}\text { Number of } \\
\text { weapons }\end{array}$ & Rate & $\begin{array}{l}\text { Number of } \\
\text { weapons }\end{array}$ & Rate \\
\hline 91 (on $1^{\text {st }}$ shot) & 76 & 83.5 & $15^{*}$ & 16.5 \\
\hline 15 (on $2^{\text {nd }}$ shot) & 11 & 73.3 & $4^{*}$ & 26.7 \\
\hline 4 (on $3^{r d}$ shot) & 3 & 75 & $1^{*}$ & 25 \\
\hline
\end{tabular}

The accomplished in-situ testing provided the client (police) with vital information. Table 2 shows that all performance parameters are within the the upper and lower performance limits established by the manufacturer when firing the weapon for three times in sequence. On the other hand, Table

Table 2. Sample test results for well-performing weapon

\begin{tabular}{|l|l|l|l|l|l|}
\hline \multirow{2}{*}{$\begin{array}{l}\text { Performance } \\
\text { parameters }\end{array}$} & \multirow{2}{*}{$\begin{array}{l}\text { Lower } \\
\text { limit }\end{array}$} & \multicolumn{3}{|l|}{ Your weapon } & \multirow{2}{*}{$\begin{array}{l}\text { Upper } \\
\text { limit }\end{array}$} \\
\cline { 3 - 6 } & & $\begin{array}{l}1^{\text {st }} \\
\text { shot }\end{array}$ & $\begin{array}{l}2^{\text {nd }} \\
\text { shot }\end{array}$ & $\begin{array}{l}3^{\text {rd }} \\
\text { shot }\end{array}$ \\
\hline PRF $(\mathrm{pps})$ & 16.5 & 18.2 & 18.2 & 18.2 & 20 \\
\hline Net charge $(\mu \mathrm{C})$ & 80 & 102 & 110 & 104 & 125 \\
\hline Pulse duration $(\mu \mathrm{s})$ & 105 & 124 & 128 & 126 & 155 \\
\hline Peak voltage $(\mathrm{V})$ & 1400 & 1400 & 1667 & 1420 & 2520 \\
\hline Peak current $(\mathrm{A})$ & 2.3 & 2.3 & 2.8 & 2.4 & 4.2 \\
\hline
\end{tabular}

3 shows the sample test results for out-of-tolerance weapon, indicating one parameter (PRF) which is beyond the limits specified by the manufacturer.

Table 3. Sample test results for out-of-tolerance weapon (Note: The out-of-tolerance measurements are marked by an asteroid.)

\begin{tabular}{|l|l|l|l|l|l|}
\hline \multirow{2}{*}{$\begin{array}{l}\text { Performance } \\
\text { parameters }\end{array}$} & \multirow{2}{*}{$\begin{array}{l}\text { Lower } \\
\text { limit }\end{array}$} & \multicolumn{3}{|l|}{ Your weapon } & \multicolumn{2}{|l|}{$\begin{array}{l}\text { Upper } \\
\text { limit }\end{array}$} \\
\cline { 3 - 6 } & & $\begin{array}{l}1^{\text {st }} \\
\text { shot }\end{array}$ & $\begin{array}{l}2^{\text {nd }} \\
\text { shot }\end{array}$ & $\begin{array}{l}3^{\text {rd }} \\
\text { shot }\end{array}$ \\
\hline PRF $(\mathrm{pps})$ & 16.5 & $15.9^{*}$ & $14.8^{*}$ & $13.4^{*}$ & 20 \\
\hline Net charge $(\mu \mathrm{C})$ & 80 & 100 & 101 & 107 & 125 \\
\hline Pulse duration $(\mu \mathrm{s})$ & 105 & 120 & 118 & 124 & 155 \\
\hline Peak voltage $(\mathrm{V})$ & 1400 & 1708 & 1719 & 1793 & 2520 \\
\hline Peak current $(\mathrm{A})$ & 2.3 & 2.9 & 2.9 & 3 & 4.2 \\
\hline
\end{tabular}

The PRF's for the first shot, second shot, and the third shot are 15.9 pps, 14.8 pps, 13.4 pps, respectively, which are below the manufacturers lower acceptable threshold of 16.5 pps.

In most of the collected pulse trains, we also measured several high and low magnitude voltage spikes at the onset of the pulse as well as where the tail of potential waveform approached to zero, see fig. 1 . This may be due to opening and closing of spark gaps in the weapon. To validate the applied 
test load as an entirely resistive load, we tested the load and measured a phase angle of 0.02 degrees, verifying a highly resistive load.

\section{CONCLUSION AND DISCUSSION}

We proposed a new in-situ TPS for CEWs which is highly portable and light, of high resolution and high accuracy with the capability of producing a manageable and small data file of several hundered kB. During field trials in the summer of 2010 with earlier equipment and a more cumbersome data file format, results from in-situ testing [2] were positive from the point of view of the police service and the academics studying the performance of CEW's. However, the test equipment was too bulky and did not lend itself to assembly and disassembly in an expeditious fashion and the memory usage was not efficient. In addition, the applied data acquisition system did not permit signal acquisition in a rapid block mode; and it needed to sample the whole stream of the input analog signal even if there was no energy present. Therefore, the aggregation of unnecessary data in the memory was the main drawback of our previous TPS. It generated a large and unmanageable data set of sampled analog signal consuming excessive amounts of computer memory, making data storage and data mining more difficult. The new TPS uses rapid block mode to only sample the desired pulses delivering energy higher than the specified trigger. Moreover, the new test system creates a new data file format for tasers involving all useful information about the weapon: taser raw data, timing data, and the applied measurement specifications in the test date - a feature absent in our earlier work. This new file format may be used for to establish a repository of electrical performance for subsequent data mining, where portions of the produced data file are utilized and processed which are of user's interest. We also hope the new file format will facilitate a collection of CEW data in a standardized form across Canada allowing research on 1) how CEW's output varies during usage and over time, 2) the variability across the population of CEWs in use, 3) out-oftolerance modes, and 4) expected lifespan of the CEW.

\section{REFERENCES}

[1] G. Breitkreuz, "Study of the conductive energy weapontaser," Report of the Standing Committee on Public Safety and National Security, Standing Order 108(2), June 2008.

[2] D. Dawson, Y. Maimaitijiang, and A. Adler, "Development of a performance calibration system for $\mathrm{x}$ 26 tasers," 2010 IEEE International Workshop on Medical Measurements and Applications Proceedings (MeMeA), 2010, pp. 107-112.

[3] SOR/98-462, "Criminal code part 3 prohibited weapons," http://www.canlii.org/en/ca/laws/regu/sor98-462/latest/sor-98-462.html (accessed Dec. 2010).

[4] A. Adler, "Workshop on conducted energy weapons, carleton university, ottawa, ontario," http://www.sce.carleton.ca/faculty/adler/talks/2009/adlerCEWWorkshop.pdf (accessed Dec. 2010), 2009.

[5] A. Adler, "Workshop on conducted energy weapons, carleton university, ottawa, ontario," http://curve.carleton.ca/papers/2010/CEW-Test-

Procedure-2010-ver1.1.pdf (accessed Dec. 2010), 2010.

[6] I.P.W. Sinclair, "Test results for the x26 conducted energy weapon (cew) in accordance with taser international device specifications," MPB Technologies - Electronics Test Centre, Report No.: A85R9006/9031/9059B1, 2009.

[7] S. Palmer, "Testing of conducted energy weapons," Canadian Police Research Centre, OPCC file 2587, 2008.

[8] P. Savard, R. Walter, and A. Dennis, "Analysis of the quality and safety of the taser $\mathrm{x} 26$ devices tested for radio-canada / canadian broadcasting corporation," National Technical Systems, Test Report 41196-08, 2008.

[9] J.R. Jauchem, "An animal model to investigate effectiveness and safety of electronic control devices (including taser devices)," J Forensic Sci., vol. 55, pp. 521-526, February 2010.

[10] A. J. Dennis, D. J. Valentino, R. J. Walter, K. K. Nagy, J. Winners, F. Bokhari, D. E. Wiley, K. T. Joseph, and R. R. Roberts, "Acute effects of taser x 26 discharges in a swine model," J. Trauma., vol. 63, pp. 581-590, 2007.

[11] A.O. Esquivel, J.A. Dawe, E.J. adn Sala-Mercado, R.L. Hammond, and C.A. Bir, "The physiologic effects of a conducted electrical weapon in swine," Ann Emerg Med., vol. 50, pp. 576-583, 2007.

[12] J. Kleinig, "Ethical constraints on taser use by police," Journal of Policy and Practice, vol. 1, pp. 284-292, 2007.

[13] A. Adler, D. P. Dawson, R. Evans, L. Garland, M. Miller, and I. Sinclair, "Toward a test protocol for conducted energy weapons," http://www.sce.carleton.ca/ adler/CEW-protocol.pdf (accessed Jan. 2011)., 2011.

[14] IEC/TS 60479-2, "Effects of current on human beings and livestock - part 2: Special aspect,” Ed. 3.0:, 2007.

[15] J. P. Reilly, A. M. Diamant, and J. Comeaux, "Dosimetry considerations for electrical stun devices," Phys. Med. Biol., vol. 54, pp. 1319-1335, 2009. 